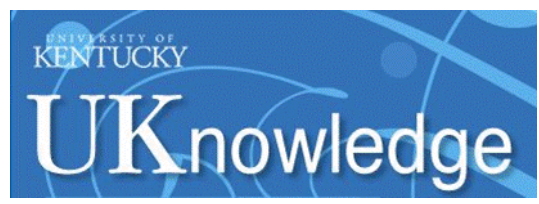

University of Kentucky

UKnowledge

$5-1-2002$

\title{
Dust Emission from Active Galactic Nuclei
}

\author{
Maia Nenkova \\ University of Kentucky \\ Željko Ivezić \\ Princeton University \\ Moshe Elitzur \\ University of Kentucky, moshe@pa.uky.edu
}

Follow this and additional works at: https://uknowledge.uky.edu/physastron_facpub

Part of the Astrophysics and Astronomy Commons, and the Physics Commons

Right click to open a feedback form in a new tab to let us know how this document benefits you.

\section{Repository Citation}

Nenkova, Maia; Ivezić, Željko; and Elitzur, Moshe, "Dust Emission from Active Galactic Nuclei" (2002). Physics and Astronomy Faculty Publications. 215.

https://uknowledge.uky.edu/physastron_facpub/215

This Article is brought to you for free and open access by the Physics and Astronomy at UKnowledge. It has been accepted for inclusion in Physics and Astronomy Faculty Publications by an authorized administrator of UKnowledge. For more information, please contact UKnowledge@lsv.uky.edu. 


\section{Dust Emission from Active Galactic Nuclei}

\section{Digital Object Identifier (DOI)}

http://dx.doi.org/10.1086/340857

\section{Notes/Citation Information}

Published in The Astrophysical Journal Letters, v. 570, no. 1, p. L9-L12.

(c) 2002. The American Astronomical Society. All rights reserved.

The copyright holder has granted permission for posting the article here. 


\title{
DUST EMISSION FROM ACTIVE GALACTIC NUCLEI
}

\author{
Maia Nenkova, ${ }^{1}$ ŽEluKo Ivezić, ${ }^{2}$ AND Moshe Elitzur ${ }^{1}$ \\ Received 2002 February 21; accepted 2002 March 26; published 2002 April 5
}

\begin{abstract}
Unified schemes of active galactic nuclei require an obscuring dusty torus around the central source, giving rise to a Seyfert 1 line spectrum for pole-on viewing and Seyfert 2 characteristics in edge-on sources. Although the observed IR is in broad agreement with this scheme, the behavior of the $10 \mu \mathrm{m}$ silicate feature and the width of the far-IR emission peak remained serious problems in all previous modeling efforts. We show that these problems find a natural explanation if the dust is contained in approximately five to 10 clouds along radial rays through the torus. The spectral energy distributions of both type 1 and type 2 sources are properly reproduced from different viewpoints of the same object if the optical depth of each cloud is $\gtrsim 60$ at visual wavelengths and if the clouds' mean free path increases roughly in proportion to the radial distance.
\end{abstract}

Subject headings: dust, extinction — galaxies: active — galaxies: nuclei — galaxies: Seyfert — quasars: general — radiative transfer

\section{INTRODUCTION}

Although there is a bewildering array of active galactic nucleus (AGN) classes, a unified scheme has been steadily emerging (e.g., Antonucci 1993, 2002; Wills 1999). The nuclear activity is powered by a supermassive $\left(\sim 10^{6}-10^{9} M_{\odot}\right)$ black hole and its accretion disk, which extends to $\sim 1 \mathrm{pc}$. This central engine is surrounded by a dusty toroidal structure, extending to $\gtrsim 100 \mathrm{pc}$. Much of the observed diversity is simply the result of viewing this axisymmetric geometry from different angles. The torus provides an anisotropic obscuration of the central region so that sources viewed face-on are recognized as Seyfert 1 galaxies and those observed edge-on are recognized Seyfert 2 galaxies. The primary evidence for the torus comes from spectropolarimetric observations of type 2 sources, which reveal hidden type 1 emission via reflection off material situated above the torus opening. While compelling, this evidence is only indirect in that it involves obscuration, not direct emission by the torus itself.

An obscuring dusty torus should reradiate in the IR the fraction of nuclear luminosity it absorbs, providing direct evidence for its existence. Indeed, the continua from most AGNs show significant IR emission. Silicates, whose presence is revealed by a spectral feature at $10 \mu \mathrm{m}$, are a major constituent of astronomical dust. Seyfert 2 sources display this feature in absorption - as expected for edge-on viewing of an optically thick torus. However, contrary to expectations for face-on viewing, quasars and Seyfert 1 galaxies do not show this feature in emission (Roche et al. 1991). ${ }^{3}$ This poses a serious problem for models of the dusty torus emission, which must suppress the silicate feature in type 1 objects while producing it in type 2 objects. Laor \& Draine (1993) studied the effects of altering the dust properties and conclude that suppression of the $10 \mu \mathrm{m}$ feature requires significant silicate depletion or very large (up to $10 \mu \mathrm{m}$ ) grains (see also Maiolino et al. 2001). This could

\footnotetext{
${ }^{1}$ Department of Physics and Astronomy, University of Kentucky, 177 Chemistry/Physics Building, Lexington, KY 40506-0055; maia@pa.uky.edu, moshe@pa.uky.edu.

${ }^{2}$ Astrophysical Sciences Department, Princeton University, Peyton Hall, Ivy Lane, Princeton, NJ 08544-1001; ivezic@astro.princeton.edu.

${ }^{3}$ Clavel et al. (2000) recently suggested that there is evidence for the $10 \mu \mathrm{m}$ emission feature in Seyfert 1 sources. However, the structure around $10 \mu \mathrm{m}$ in the composite spectrum that they present is neither as pronounced nor as broad as the standard silicate feature.
}

explain type 1 spectral energy distributions (SEDs) but would require a different explanation for type 2 objects with their prominent $10 \mu \mathrm{m}$ absorption features.

Pier \& Krolik $(1992,1993)$ were the first to explore the effects of toroidal geometry on dust radiative transfer. They note that the AGN dust must be in clumps to protect the grains, but because of the difficulties in modeling a clumpy distribution, they approximate it with a uniform one instead. They also neglect scattering. In spite of these approximations, their results are encouraging. The directional dependence of their model radiation reproduces the gross features of observed SEDs, indicating that the toroidal geometry captures the essence of IR observations. Two major problems remain: The observed far-IR emission covers a much broader range than the models can produce, and the silicate emission feature is suppressed in face-on viewing only in a narrow, finely-tuned range of the model parameters (see also Granato \& Danese 1994 and Granato, Danese, \& Franceschini 1997 for models of extended tori). Rowan-Robinson (1995) notes that both problems could be alleviated by clumpiness. He argues that a thin spherical shell around a central radiation source is an adequate approximation for a small clump illuminated by that source at a large distance, and produces type 1 IR emission from a superposition of such concentric shells. But this shell/clump analogy is fundamentally problematic: A clump emission is highly anisotropic because the illuminated and dark sides of a clump have widely different temperatures. In contrast, the emission from a spherical shell surrounding the source is isotropic. The strong variation of clump SEDs with angle between radiation source, clump, and observer (see Fig. 1) cannot be reproduced by the spherical shell.

We performed a more adequate calculation of IR emission from an obscuring clumpy torus, combining realistic modeling of the emission from an externally illuminated clump with proper handling of cloud shadowing. The latter is an essential ingredient since the number of clouds along radial rays through the torus must be sufficiently large to ensure that X-rays from the central engine are virtually always attenuated in type 2 objects (Guainazzi et al. 2001). Here we report the results of our effort, which show that the SEDs of both type 1 and type 2 objects are properly reproduced at different viewing angles of the same clumpy torus-in agreement with unified schemes. 

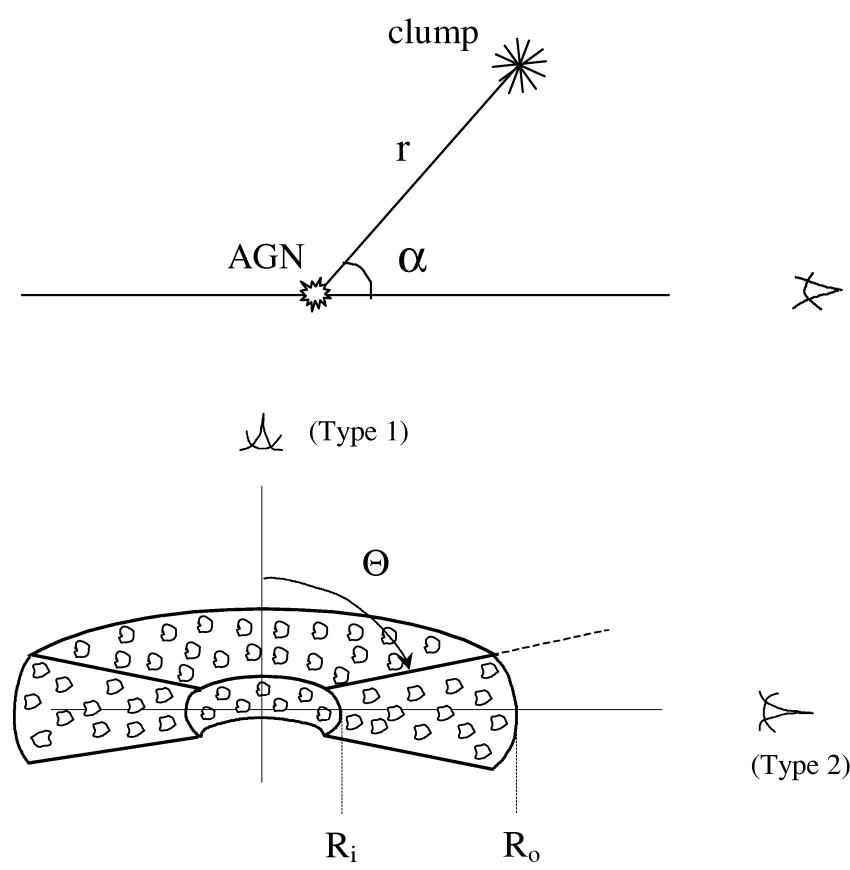

(Type 2)

FIG. 1.-Model geometry. Top: Positions of the AGN, clump, and observer. As the position angle $\alpha$ varies at fixed distance $r$, the visible fraction of the clump's illuminated area changes (like phases of the Moon) and with it the observed radiation. Bottom: Clumpy torus.

In a forthcoming publication, we will provide a more detailed account of our calculations and the results.

\section{EMISSION FROM A CLUMPY MEDIUM}

Let us consider a medium where the dust is in clouds. For simplicity, each cloud has the same optical depth $\tau_{\lambda}$. Along a given path, the mean number of clouds encountered in segment $d s$ is $d N(s)=d s / l(s)$, where $l=\left(n_{c} A_{c}\right)^{-1}$ is the mean free path between clouds ( $A_{c}$ is the cloud area perpendicular to the path, and $n_{c}$ is the number density of clouds). As long as $l \gg R_{c}$, where $R_{c}$ is the cloud radius, each cloud can be considered a point source of intensity $S_{c \lambda}$, and the intensity generated along the segment is $S_{c \lambda} d N$. Let us denote by $P_{\text {esc }}$ the probability that this radiation propagates along the rest of the path without absorption by any other cloud. Natta \& Panagia (1984) show that Poisson statistics for the distribution of clumps yields

$$
P_{\mathrm{esc}}=e^{-t_{\lambda}},
$$

where $t_{\lambda}=N(s)\left(1-\epsilon^{-\tau_{\lambda}}\right)$ and $N(s)=\int_{s} d N$ is the mean number of clouds along the rest of the path. The contribution of segment $d s$ to the emerging intensity is simply $P_{\text {esc }} S_{c \lambda} d N$, and the flux from the cloud distribution at distance $D$ is

$$
F_{\lambda}^{C}=\frac{1}{D^{2}} \int d A \int e^{-t \lambda} S_{c \lambda}(s) d N(s)
$$

where $d A$ is the surface area element perpendicular to the line of sight. Given the geometry, the flux can be calculated from this expression once $S_{c \lambda}$ is known.

Clouds are heated by radiation from both the AGN and all other clouds. Let us consider first the direct heating by the AGN (Fig. 1). Since our interest is in optically thick clouds only, the dust temperature is much higher on the illuminated side than any other part of the surface. Therefore, the cloud emission varies strongly with direction, and the corresponding source function $S_{c \lambda}^{d}(r, \alpha)$ depends on both distance $r$ and position angle $\alpha$. The clump shape is of course arbitrary, and we have constructed "synthetic clumps" by averaging the emission from an illuminated slab over all possible slab orientations. This procedure utilizes an exact solution of radiative transfer for external illumination and also reproduces the $\alpha$-dependence of the observed fraction of illuminated area on the surface of a spherical-like object.

We conducted detailed calculations with the code DUSTY (Ivezić, Nenkova, \& Elitzur 1999), ${ }^{4}$ which preforms an exact solution of the slab radiative transfer problem including dust absorption, emission, and scattering. The optical depth across the slab thickness at wavelength $\lambda$ is $\tau_{\lambda}=q_{\lambda} \tau_{V}$, where $\tau_{V}$ is the optical depth at visual and $q_{\lambda}$ is the proper efficiency factor of standard interstellar dust. With AGN luminosity $L_{12}=$ $L / 10^{12} L_{\odot}$, the illuminating bolometric flux is $F_{e}=L / 4 \pi r^{2}$. Its spectral shape, typical for AGNs, is $\lambda f_{\lambda}=$ constant in the wavelength range of $0.01-0.1 \mu \mathrm{m}$ and is proportional to $\lambda^{-0.5}$ for 0.1-100 $\mu \mathrm{m}$ (see Pier \& Krolik 1992 and Laor \& Draine 1993). For any slab orientation, the solution of the radiative transfer problem determines the temperature run in the slab and its emission in any direction. Therefore, the dust temperature $T_{n}$ on the illuminated face of a slab normal to the radius vector can replace the external flux $F_{e}$ as a specifier of location.

The top panel of Figure 2 shows typical results for $S_{c \lambda}^{d}$. The clump spectrum was constructed by averaging over all slab orientations the solutions for slabs with $\tau_{V}=100$ at radial distances where $T_{n}=800 \mathrm{~K}$ (corresponding to $r=4 L_{12}^{1 / 2} \mathrm{pc}$ ). The $10 \mu \mathrm{m}$ silicate feature is seen in absorption at small $\alpha$, switching to increasingly more pronounced emission as $\alpha$ increases and a larger fraction of the clump's illuminated face becomes visible.

An exact calculation of the effects of diffuse radiation from all clouds is a formidable task, akin to a full solution of the radiative transfer problem in which individual dust particles themselves are dusty clouds. However, compared with the AGN radiation, heating by the diffuse radiation is highly inefficient because it involves long wavelengths and thus can be neglected. Emission from clouds with a direct view of the AGN is well described by $S_{c \lambda}^{d}$. But clouds whose line of sight to the AGN is blocked by another cloud will be heated only indirectly by the diffuse radiation. We approximate the diffuse radiation field at $r$ by angle-averaging over $\alpha$ the emission $S_{c \lambda}^{d}(r, \alpha)$ from clouds directly heated by the AGN. In this radiation field, we embed a sphere with a constant density profile and solve for its temperature and emission. Our approximation for the source function of indirectly illuminated clouds is $S_{c \lambda}^{i}=F / \Omega$, where $F$ is the flux and $\Omega$ the solid angle of such a sphere at a large distance. The bottom panel of Figure 2 shows a sample of $S_{c \lambda}^{i}$, together with the corresponding source functions for direct heating. At distance $r$, the probability for an unhindered view of the AGN is $p(r)=e^{-N(r)}$, where $N(r)=\int^{r} d r / l$ is the mean number of clouds to the AGN. Our approximation for the clump source function is

$$
S_{c \lambda}=p S_{c \lambda}^{d}+(1-p) S_{c \lambda}^{i}
$$

The steps that we outlined can be iterated to become an exact

\footnotetext{
${ }^{4}$ Accessible at http://www.pa.uky.edu/ moshe/dusty.
} 


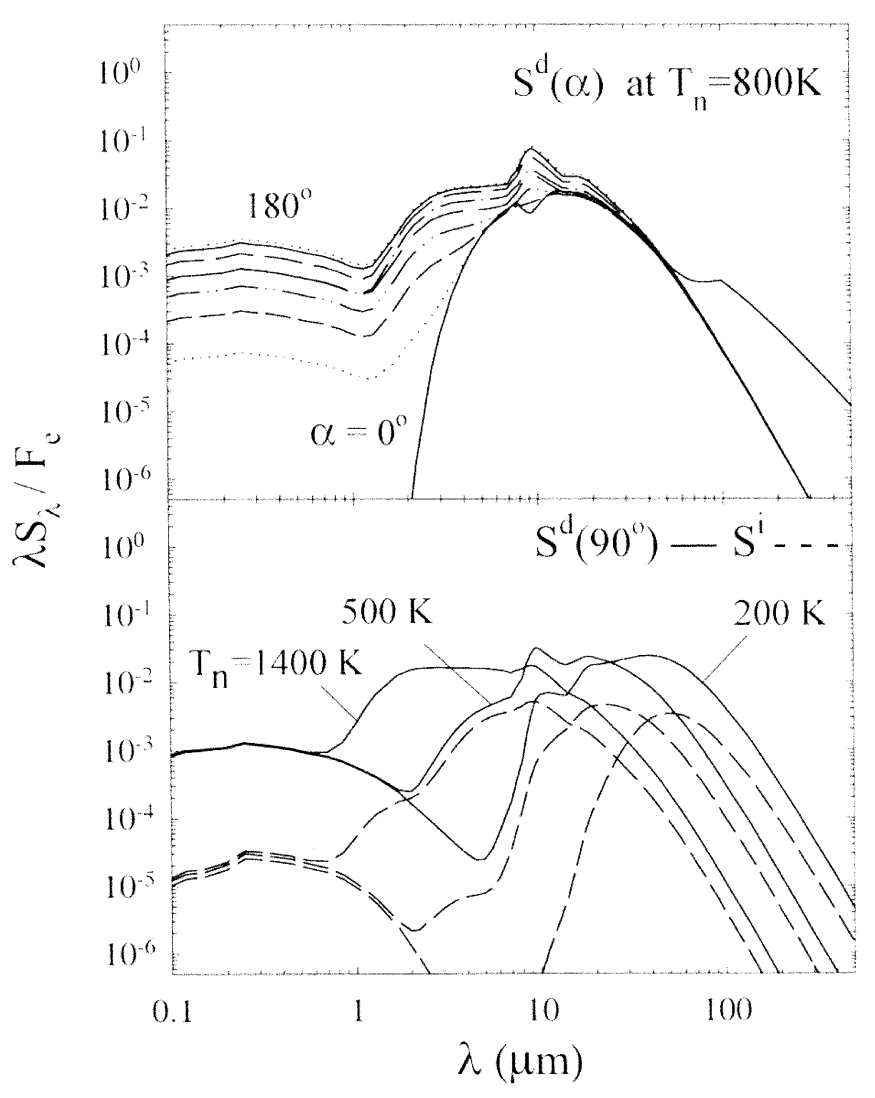

FIG. 2.-Emission from $\tau_{V}=100$ clumps normalized to the AGN local bolometric flux $F_{e}=L / 4 \pi r^{2}$. Top: Source $S_{c \lambda}^{d}$ for directly heated clouds at a radial distance where $T_{n}=800 \mathrm{~K}\left(r=4 L_{12}^{1 / 2} \mathrm{pc}\right.$ for this $\left.\tau_{V}\right)$. The position angle $\alpha$ is shown at $22^{\circ} .5$ intervals. Bottom: Emission of directly ( $S_{c \lambda}^{d}$, solid lines) and indirectly $\left(S_{c \lambda}^{i}\right.$, dashed lines $)$ heated clouds at distances where $T_{n}=$ 1400,500 , and $200 \mathrm{~K}$, or $r / L_{12}^{1 / 2}=1,10$, and $100 \mathrm{pc}$, respectively. The source function for direct heating is shown at $\alpha=90^{\circ}$.

solution scheme for radiative transfer in clumpy media. The small magnitude of $S_{c \lambda}^{i} / S_{c \lambda}^{d}$ at short wavelengths, which control heating, evident in Figure 2 indicates that this process should converge rapidly. Equation (3) is equivalent to its first two steps.

\section{MODEL RESULTS}

We model the AGN-obscuring region as a toroidal distribution of dusty clouds, shown in Figure 1. Instead of the inner radius $R_{i}$, we specify as input $T_{n, i}=T_{n}\left(R_{i}\right)$, the surface temperature of a normally illuminated slab at $R_{i}$. In all calculations, we set $T_{n, i}=1400 \mathrm{~K}$, the temperature below which both silicate and graphite grains exist; this choice implies $R_{i}=$ $1.2 L_{12}^{1 / 2}$ pc for a $\tau_{V}=100$ cloud. Geometry input parameters are the torus thickness $Y=R_{o} / R_{i}$, equivalent to the lowest $T_{n}$ in the torus, and $\Theta$, the conical opening half-angle. Assuming a power-law variation for the mean free path $l \propto r^{q}$, the cloud distribution is described by the two input parameters $q$ and $N_{T}=\int_{R_{i}}^{R_{o}} d r / l$, the number of clouds along a radial ray through the torus. The final free parameter is $\tau_{V}$, the optical depth of each cloud. The grain efficiency factor $q_{\lambda}$ and the AGN spectral shape $f_{\lambda}$ are set to their standard values. The observed flux is determined for type 1 sources from $F_{\lambda}=\left(L / 4 \pi D^{2}\right) f_{\lambda}+F_{\lambda}^{C}$, where $F_{\lambda}^{C}$ is from equation (2). For type 2 sources, the first term is omitted since even a single cloud along the line of sight would block the AGN completely. Extracting the overall bo-

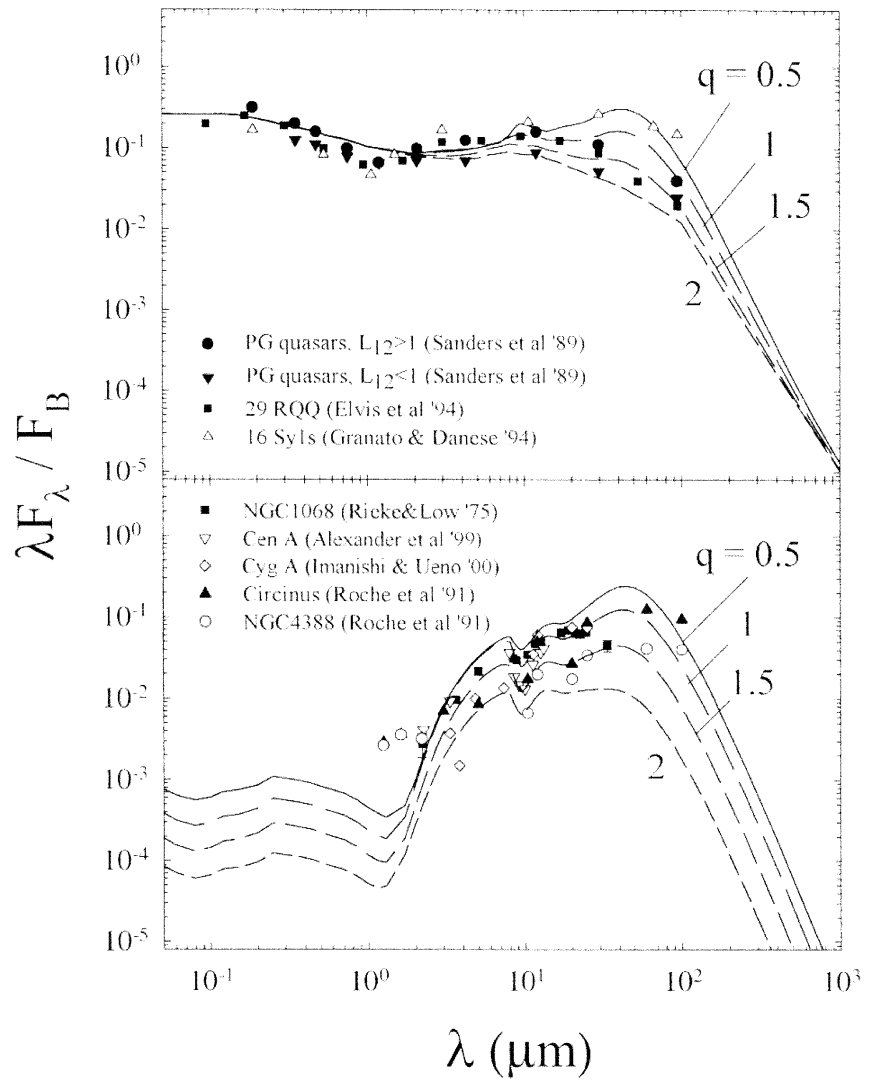

Fig. 3.-Modeling and observations of type 1 (top) and type 2 (bottom) sources. The lines are model results for axial and equatorial views of the clumpy torus shown in Fig. 1, with $F_{B}=L / 4 \pi D^{2}$. Radial rays through the torus have five clouds on average, each with $\tau_{V}=100$. The clouds' mean free path varies as $r^{q}$, with the indicated $q$. The torus inner radius corresponds to temperature $T_{n, i}=1400 \mathrm{~K}\left(R_{i}=1.2 L_{12}^{1 / 2} \mathrm{pc}\right)$, and it has $R_{o}=100 R_{i}$ and $\Theta=45^{\circ}$. Data for type 1 are average spectra from the indicated compilations; data for type 2 are for individual objects. Each data set was scaled for a rough match with the models.

lometric flux $F_{B}=L / 4 \pi D^{2}$, the SED is controlled by $T_{n, i}$ and five dimensionless free parameters: $Y$ and $\Theta$ specify the geometry, $\tau_{V}$ the individual clouds, and $\mathrm{N}_{T}$ and $q$ the cloud distribution.

We performed extensive calculations and found a large range of the free parameters that yield satisfactory agreement with observations. A detailed report of our results will be provided elsewhere; here we show in Figure 3 a representative model. Our aim is to reproduce the typical SED of the type 1 and type 2 families, not to fit in detail any particular object. Data points for type 1 sources show average spectra for radio-quiet quasars and Seyfert 1 galaxies. Because of the high obscuration of the AGNs in type 2 sources, fluxes for their nuclear regions properly extracted out of the contributions of the host galaxy and starburst regions are scarce. We show data for reliable measurements of five individual objects. The data were scaled for a rough match of the model results without attempting a best fit. Detailed fitting of type 2 objects, when warranted, will determine their bolometric flux $F_{B}$, a quantity inaccessible for direct measurement because the bulk of the flux is emitted away from Earth. The strong $\sim 1-2 \mu \mathrm{m}$ emission in some sources could indicate the contribution from clouds above the torus opening.

Our models reproduce the broad IR bump extending to 
$\sim 100 \mu \mathrm{m}$, as observed. Furthermore, the $10 \mu \mathrm{m}$ feature appears in absorption in equatorial viewing but is smeared out in axial viewing in spite of its prominence in emission from directly heated individual clouds (Fig. 2). We find satisfactory results for a conical opening $\Theta=45^{\circ} \pm 15^{\circ}$. The torus thickness can vary in the range $Y=50-250$; i.e., $R_{o} / L_{12}^{1 / 2}=60-300$ pc. At smaller $Y$, the IR bump is too narrow; larger $Y$ produce too much far-IR emission. The number of clouds is $N_{T}=5-10$. When $N_{T}<5$, the $10 \mu \mathrm{m}$ feature appears in emission in poleon spectra; $N_{T}>10$ shifts the spectrum too far to long wavelengths. The power index should be $q=1 \pm 0.5$ (see Fig. 3). Significantly, the only constraint on the optical depth of individual clouds is $\tau_{V} \gtrless 60$. The results vary only slightly when $\tau_{V}$ increases from 60 to 100 and hardly at all during further increases. The reason is simple. The dependence on $\tau_{V}$ arises from the probability of photon escape and the cloud source function. From equation (1), $P_{\mathrm{esc}}=e^{-N}$ whenever $\tau_{\lambda} \gg 1$, and at $\tau_{V} \geq 60$, this condition is met at all relevant wavelengths. Similarly, because each cloud is heated from outside, only its surface is heated significantly when $\tau_{V}$ is large. Increasing $\tau_{V}$ further only adds cool material; thus, $S_{c \lambda}$ saturates for all relevant $\lambda$ (similar to standard blackbody emission). Extending our calculations all the way to $\tau_{V}=500$, we have verified that increasing $\tau_{V}$ indeed has no effect on the model results.

\section{DISCUSSION}

The cloud distribution is described by $q$ and $N_{T}$, and individual clouds are described by the optical depth $\tau_{v}$. No other cloud property was specified. The cloud size enters only indirectly through the underlying assumption $R_{c} \ll l$. If $V_{c} \simeq$ $A_{c} R_{c}$ is the volume of a single cloud, the volume filling factor of the cloud population is $\phi=n_{c} V_{c}=R_{c} / l$. Our calculations apply to small filling factors, $\phi \ll 1$; the Pier \& Krolik model involves the opposite limit $\phi=1$. The mean free path scale can be determined from $l\left(R_{i}\right) / R_{i}=\left(1 / N_{T}\right) \int_{1}^{Y} y^{-q} d y$. The model with $q=1, N_{T}=5$, and $Y=100$ has $l\left(R_{i}\right)=0.9 R_{i}=$ $1.1 L_{12}^{1 / 2} \mathrm{pc}$. A reasonable realization of this model, although not unique, is a constant $\phi=0.1$ throughout the torus, so that
$R_{c}=0.09 r$. When $\tau_{V}=100$ and $L_{12}=1$, the clouds vary from $R_{c} \sim 0.1 \mathrm{pc}$ with gas density $\sim 3 \times 10^{5} \mathrm{~cm}^{-3}$ in the torus inner regions to $R_{c} \sim 10 \mathrm{pc}$ with density $\sim 3 \times 10^{3} \mathrm{~cm}^{-3}$ at the outer edge. The torus could contain additional clouds with smaller $\tau_{V}$ without significantly affecting the SED.

The properties that we deduce for the torus agree with independent estimates. Based on 88 Seyfert galaxies, Schmitt et al. (2001) conclude that $\Theta=48^{\circ}$, at the center of the range that we found. Our minimal cloud number $N_{T}=5$ implies a probability $\leq e^{-5}=7 \times 10^{-3}$ for an unattenuated view of the AGN in type 2 sources, in agreement with the findings of Guainazzi et al. (2001). From X-ray measurements of 73 Seyfert 2 galaxies, Bassani et al. (1999) find a large variation in column density, with a mean of $3 \times 10^{23} \mathrm{~cm}^{-2}$ and $\geqslant 10^{24} \mathrm{~cm}^{-2}$ for as many as a third of the sources. This mean is comparable to the column density of our torus for a standard dust-to-gas ratio and five clouds of $\tau_{V} \geqslant 60$ each. Furthermore, a natural consequence of our model is the observed similarity of SEDs among type 2 sources in spite of the large X-ray column variation; since $\tau_{V}$ is bounded only from below, the SED remains the same at all $\lambda \gtrsim 1 \mu \mathrm{m}$ for an arbitrary increase in the overall column. In contrast, the X-ray absorption does vary with $\tau_{V}$ because the optical depth for Thomson scattering is only $\sim 10^{-2} \tau_{v}$. Sources with small columns may show up in X-ray absorption while being selectively excluded from IR observations because of their weak emission.

Our results add strong support to unification schemes of AGNs. In accordance with such schemes, the IR emission from both type 1 and type 2 sources is reproduced at different viewings of the same geometry. Proper account of the torus clumpiness (1) resolves the $10 \mu \mathrm{m}$ silicate feature problem, (2) removes the difficulties in reproducing the full range of observed IR wavelengths, and (3) explains the similarity of SEDs among sources whose X-ray observations indicate widely different column densities.

We have greatly benefited from discussions with many colleagues, especially J. Conway, A. Laor, N. Levenson, and H. Netzer. Support by NASA and NSF is gratefully acknowledged.

\section{REFERENCES}

Alexander, D. M., Efstathiou, A. Hough, J. H., Aitken, D. K., Lutz, D., Roche, P. F., \& Sturm, E. 1999, MNRAS, 310, 78

Antonucci, R. 1993, ARA\&A, 31, 473

- 2002, in Astrophysical Spectropolarimetry, ed. J. Trujillo-Bueno, F.

Moreno-Insertis, \& F. Sanchez (Cambridge: Cambridge Univ. Press), 151

Bassani, L., Dadina, M., Maiolino, R., Salvati, M., Risaliti, G., della Ceca,

R., Matt, G., \& Zamorani, G. 1999, ApJS, 121, 473

Clavel, J., et al. 2000, A\&A, 357, 839

Elvis, M., et al. 1994, ApJS, 95, 1

Granato, G. L., \& Danese, L. 1994, MNRAS, 268, 235

Granato, G. L., Danese, L., \& Franceschini, A. 1997, ApJ, 486, 147

Guainazzi, M., Fiore, F., Matt, G., \& Perola, G. C. 2001, MNRAS, 327, 323

Imanishi, M., \& Ueno, S. 2000, ApJ, 535, 626

Ivezić, Ž., Nenkova, M., \& Elitzur, M. 1999, User Manual for DUSTY, Univ. Kentucky Internal Rep.

Laor, A., \& Draine, B. T. 1993, ApJ, 402, 441
Maiolino, R., Marconi, A., Salvati, M., Risaliti, G., Severgnini, P., Oliva, E., La Franca, F., \& Vanzi, L. 2001, A\&A, 365, 28

Natta, A., \& Panagia, N. 1984, ApJ, 287, 228

Pier, E., \& Krolik, J. 1992, ApJ, 401, 99 1993, ApJ, 418, 673

Rieke, G., \& Low, F. 1975, ApJ, 199, L13

Roche, P. F., Aitken, D. K., Smith, C. H., \& Ward, M. J. 1991, MNRAS, 248, 606

Rowan-Robinson, M. 1995, MNRAS, 272, 737

Sanders, D. B., Phinney, E. S., Neugebauer, G., Soifer, B. T., \& Matthews, K. 1989, ApJ, 347, 29

Schmitt, H. R., Antonucci, R. R. J., Ulvestad, J. S., Kinney, A. L., Clarke, C. J., \& Pringle, J. E. 2001, ApJ, 555, 663

Wills, B. J. 1999, in ASP Conf. Ser. 162, Quasars and Cosmology, ed. G. Ferland \& J. Baldwin (San Francisco: ASP), 101 